\title{
Right ventricular outflow tract obstruction secondary to small intestinal lymphoma
}

\author{
I D A JOHNSTON, A W POPPLE \\ From the Departments of Cardiology and Pathology, St. George's Hospital, London
}

SUMMARY A 71-year-old man developed symptoms and signs of right ventricular outflow obstruction. After angiography, which showed multiple lobulated and well-defined filling defects in the right ventricular outflow tract, endomyocardial biopsy disclosed infiltration of the myocardium by a poorly differentiated malignant neoplasm. At necropsy he was found to have a small intestinal lymphoma.

\section{Case report}

A 71-year-old man was admitted with a sevenmonth history of progressive exertional dyspnoea, extreme tiredness, anorexia, and sweats. There was no relevant past history and he had been on no medication.

On examination, he was pale but not clubbed and he had no lymphadenopathy. The heart rate was normal and the blood pressure $140 / 80 \mathrm{mmHg}$. The central venous pressure was raised $10 \mathrm{~cm}$ above the sternal angle, with a prominent a wave, and the apex beat was displaced to the anterior axillary line in the sixth intercostal space. Auscultation showed an early diastolic sound which was either a delayed pulmonary component of the second sound or a right ventricular third sound. There was a grade $4 / 6$ ejection systolic murmur maximal at the pulmonary area. There was a trace of ankle oedema and the liver was palpable $3 \mathrm{~cm}$ below the costal margin and was of normal consistency and not tender. The spleen was impalpable and examination was otherwise unremarkable.

\section{INVESTIGATIONS}

The haemoglobin was $10.9 \mathrm{~g} / \mathrm{dl}$ with MCV $76 \mathrm{fl}$. and a mildly hypochromic film. The white count was $8500 / \mathrm{mm}^{3}$ with a norma Jdifferential and the erythrocyte sedimentation rate was $73 \mathrm{~mm}$ in one hour. The alkaline phosphatase and alanine transaminase were both marginally raised, but routine biochemical screen was otherwise normal, apart from a low iron and iron binding capacity. Protein electrophoresis showed an increased $\alpha-2$ globulin.
Sputum culture and cytology were negative. Bonemarrow aspiration showed reticuloendothelial iron block with anaemia, probably secondary to malignancy or infection.

The chest $x$-ray film (Fig. 1a) showed an enlarged heart with a prominent bulge on the upper left heart border. There was pleural shadowing at the left base and bullae in both lung fields. Mediastinal tomography showed a soft tissue shadow in the region of the right ventricular outflow tract, with a convex outer border. It was not possible to say whether this represented an intrinsic lesion of the heart itself or a mass outside the heart. Bronchoscopy was normal. The electrocardiogram showed left axis deviation and non-specific ST changes in the lateral chest leads. There was no evidence of right ventricular hypertrophy or right bundle-branch block.

The routine echocardiogram showed normal left ventricular cavity size, but the ventricular wall was hypokinetic. The interventricular septum was disproportionately thickened, and there were prominent echoes from the anterior heart wall, but with normal right ventricular cavity size. Using simultaneous high-speed echocardiography and phonocardiography, the early diastolic sound noted on auscultation was shown to coincide with pulmonary valve closure, which was thus shown as being 0.08 seconds after aortic closure in expiration. These findings were consistent with right ventricular outflow tract obstruction, but even in retrospect there was no evidence from the echocardiogram of a space-occupying lesion.

Cardiac catheterisation showed a gradient of 

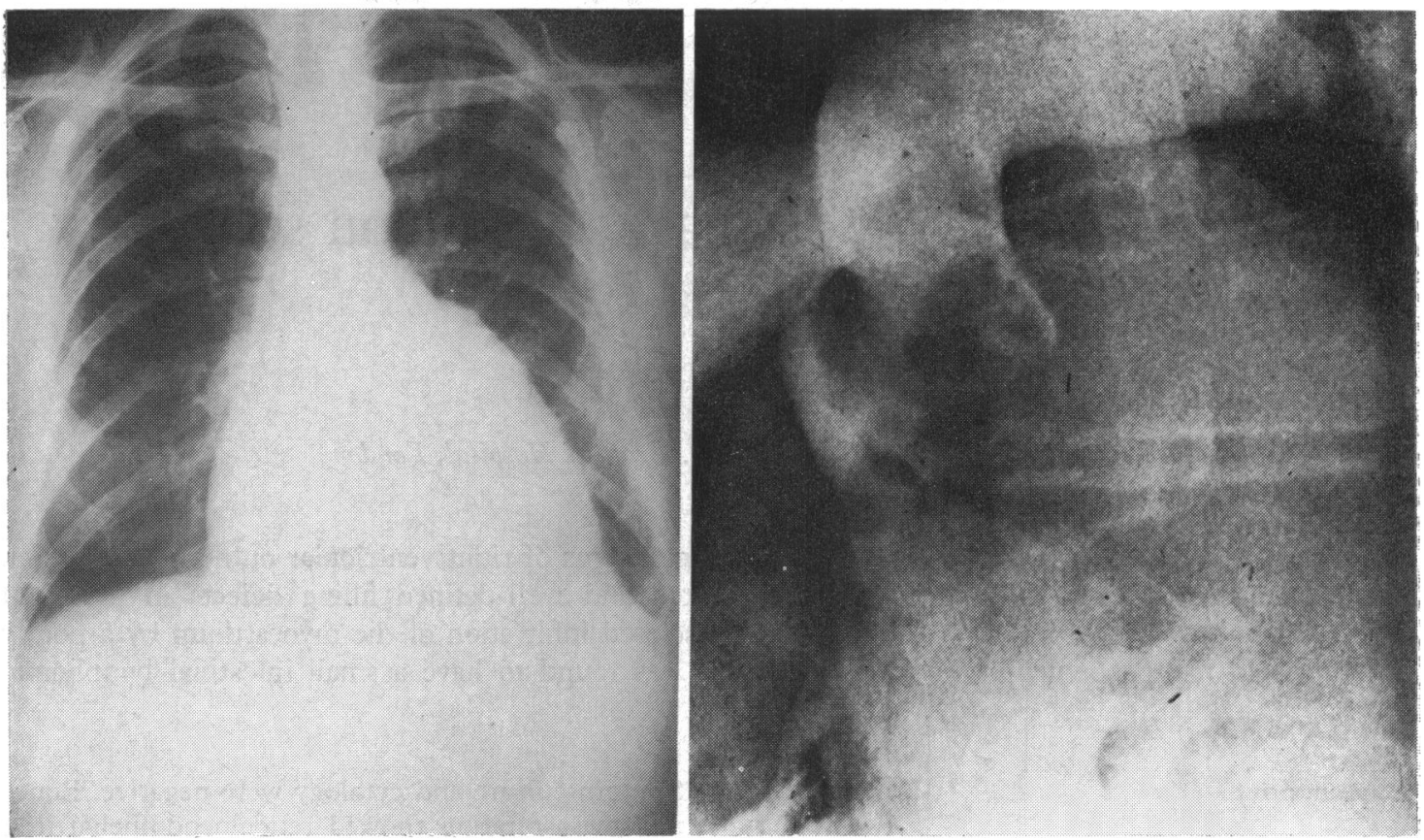

Fig. 1 (a) Chest x-ray film showing an enlarged heart with a prominent bulge on the upper left heart border. (b) Right ventricular angiogram (oblique view), showing multiple lobulated filling defects in the outflow tract.

$55 \mathrm{mmHg}$ across the right ventricular outflow tract, and angiography (Fig. 1b) showed multiple lobulated and well-defined filling defects in the right ventricular outflow tract which prolapsed into the main pulmonary artery during ventricular ejection.

\section{SUBSEQUENT COURSE}

The patient's condition steadily worsened after his admission, and he developed atrial arrhythmias. Surgical resection of the tumour was considered, and he thus underwent endomyocardial biopsy, using the technique described by Brooksby et al. ${ }^{1}$ Though this procedure did not appear directly to affect the patient adversely, he continued to deteriorate over the subsequent week, becoming hypotensive with further arrhythmias, and died.

\section{PATHOLOGY}

The cardiac biopsy comprised five fragments of tissue. These showed infiltration of the myocardium by a poorly differentiated malignant neoplasm (Fig. 2a), the appearances of which were consistent with a malignant lymphoma or a poorly differentiated secondary carcinoma.

At necropsy, tumour was found infiltrating the right ventricular outflow tract, extending from $3 \mathrm{~cm}$ below the pulmonary valve to the bifurcation of the pulmonary artery (Fig. 2b). Only one cusp of the pulmonary valve was spared. Tumour was seen in the wall of the left atrium and in the anterior part of the wall of the left ventricle, but it did not encroach into either chamber.

Tumour was also seen in hilar and mesenteric lymph nodes, and in the small bowel. The multifocal nature of the tumour deposits in the small bowel mucosa is characteristic of a malignant lymphoma. Furthermore, such an appearance is highly suggestive of local origin of the lymphoma in the bowel, so that the cardiac deposits must be regarded as metastases. The liver was congested but contained no secondary deposits.

The histological appearances confirmed a malignant lymphoma of the poorly differentiated lymphocytic type.

\section{Discussion}

After several necropsy series the incidence of cardiac metastasis in patients with lymphoma is now known to be much higher than was previously thought. An incidence of 24 per cent was found ${ }^{2}$ in 196 patients with a malignant lymphoma studied at necropsy, though the incidence of symptoms caused by cardiac involvement is probably less than 10 per cent of those with metastases. The 


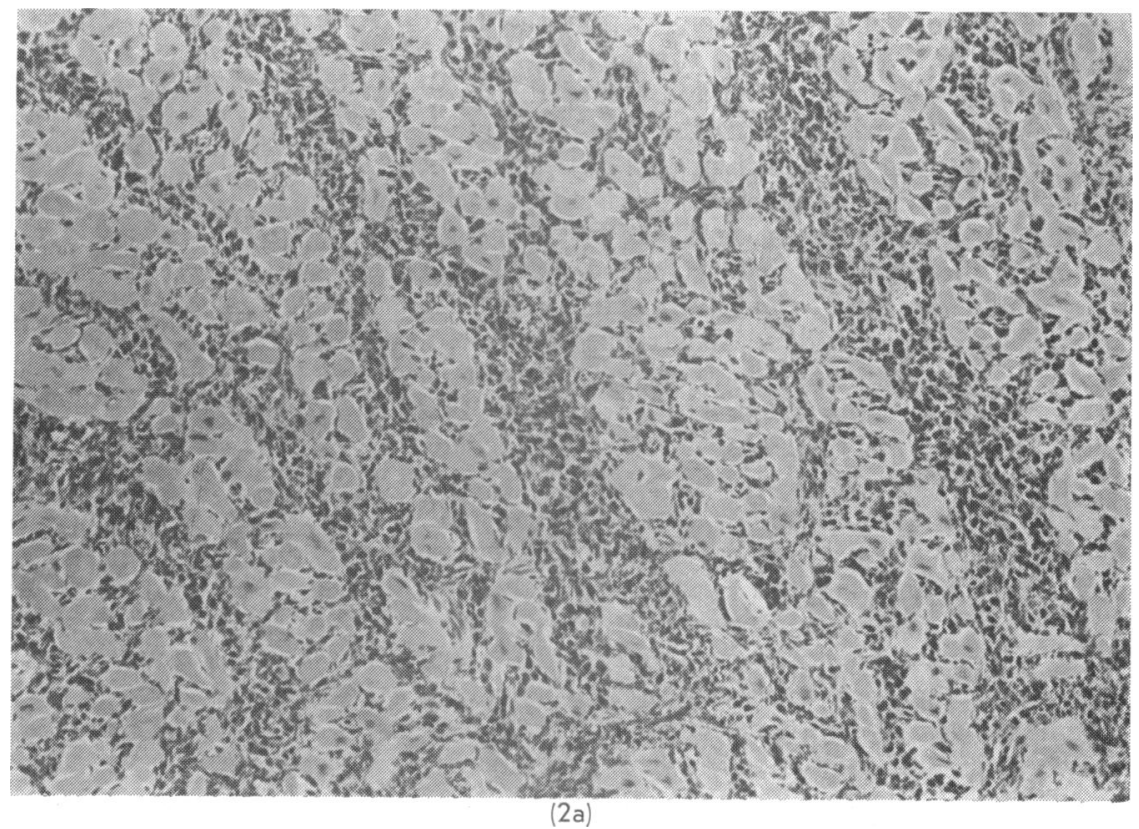

Fig. 2 (a) Cardiac biopsy; myocardial fibres infiltrated by a poorly differentiated malignant neoplasm. (Haematoxylin and eosin. $\times 20$ \%).) (b) L.obulated white tumour masses fill the right ventricular outflow tract, and the surrounding myocardium is thickened with tumour.

most common site of involvement is the pericardlum, and endocardial lesions are rare. Thus the usual form of presentation is with pericardial effusion or arrhythmias, often atrial fibrillation. Cardiac obstruction and embolism, common symptoms of primary heart tumours, are less common with metastases which tend to be invasive without producing space-occupying lesions. ${ }^{3}$

It is also rare for the cardiac manifestation to be the presenting feature in lymphoma, and we have been unable to find a similar case of a deposit from an occult small intestinal lymphoma producing symptomatic right ventricular outflow obstruction. Such a presentation, as indicated above, is well recognised in primary heart tumours, both rhabdomyosarcomata ${ }^{4}$ and myxomata. ${ }^{6}$

The diagnosis of cardiac tumours may be difficult because of their extremely variable manifestations, but it is important in view of the possibility of treatment. Echocardiography has been shown to be of great value in the diagnosis, particularly of atrial myxomata, but also of right ventricular tumours, ${ }^{7}$ though it should be noted that the echocardiograms reported by Ports et al. were initially interpreted wrongly, and only retrospectively was it shown that a correct diagnosis could have been made. In our case, combined echophonocardiography suggested that there was right ventricular outflow obstruction, but there

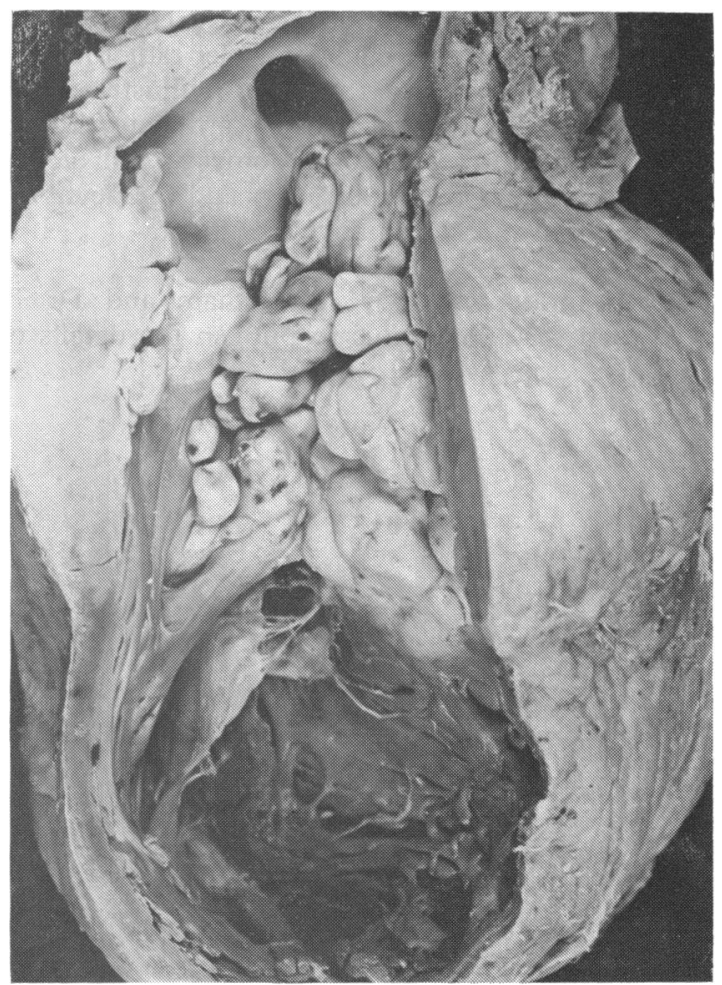

(2b) 
were no abnormal echoes from within the right ventricular cavity to suggest a tumour.

Here the technique of endomyocardial biopsy was valuable. Firstly, it indicated that the tumour was invading the myocardium and was thus not amenable to a surgical approach. This is, fortunately, not the case if a myxoma is found, and Snyder et al., ${ }^{8}$ reviewing the published reports, stated that 11 out of 13 patients with right ventricular myxoma survived operation and no recurrence was noted, whereas other tumours had a poor prognosis. Secondly, the biopsy suggested lymphoma. The results of several studies clearly indicate the value of radiotherapy in cardiac lymphoma, both in terms of symptomatic improvement and, to some extent, prolonged survival. Radiotherapy can reverse arrhythmias and control pericardial effusions. Cham et $a l .{ }^{9}$ reported improvement in six out of seven patients for between two and four months; Terry and Kligerman ${ }^{10}$ described seven patients of whom one was alive without evidence of the disease at two years, and another at one year. Radiotherapy was also used successfully by Liedtke et $a l .{ }^{11}$ in a case with secondary lymphoma obstructing the pulmonary valve and proximal pulmonary artery, with the patient continuing to improve over an eight-month follow-up period. Unfortunately, our patient's general condition was extremely poor, and he died before we could consider radiotherapy.

Despite the rarity of this case, it illustrates the need for a definitive diagnosis of cardiac tumour to be made, and the value of endomyocardial biopsy in establishing both the diagnosis and the possibilities of curative and palliative treatment.

We are grateful to $\mathrm{Dr} A$ Leatham and Dr D Redwood for allowing us to publish the details of this case.

\section{References}

${ }^{1}$ Brooksby IAB, Swanton RH, Jenkins BS, WebbPeploe MM. Long sheath technique for introduction of catheter tip manometer as endomyocardial bioptome into left or right heart. $\mathrm{Br}$ Heart $\mathcal{f} 1974$; 36: 908-12. ${ }^{2}$ Roberts WC, Glancy DL, DeVita VT Jr. Heart in malignant lymphoma (Hodgkins disease, lymphosarcoma, reticulum cell sarcoma and mycosis fungoides). Am f Cardiol 1968; 22: 85-107.

${ }^{3}$ Goodwin JF. The spectrum of cardiac tumours. $A m \mathcal{F}$ Cardiol 1968; 21: 307-14.

${ }^{4}$ Hallerman FJ, Kincaid OW, Brown AL, Daugherty GW. Rhabdomyosarcoma of the heart producing right ventricular outflow obstruction. $\mathcal{F} A M A 1963 ; 184$ : 939-42.

${ }^{5}$ Pund EE Jr, Collier TM, Cunningham JE Jr, Hayes JR. Primary cardiac rhabdomyosarcoma presenting as pulmonary stenosis. Am f Cardiol 1963; 12: 249-53.

${ }^{6}$ Gottesgen G, Wessely J, Array A, Temesvari A. Right ventricular myxoma simulating pulmonic stenosis. Circulation 1963; 27: 95-7.

'Ports TA, Schiller NB, Strunk BL. Echocardiography of right ventricular tumours. Circulation 1977; 56: 439-47.

${ }^{8}$ Snyder SN, Smith DC, Lau FYK, Turner AF. Diagnostic features of right ventricular myxoma. $\mathrm{Am}$ Heart f 1976; 91 : 240-48.

${ }^{9}$ Cham WC, Freiman AH, Carstens PHB, Chu $\mathrm{FCH}$. Radiation therapy of cardiac and pericardial metastases. Radiology 1975; 114: 701-4.

${ }^{10}$ Terry LN Jr, Kligerman MM. Pericardial and myocardial involvement by lymphomas and leukemias. Cancer 1970; 25: 1003-8.

${ }^{11}$ Liedtke AJ, Adams DF, Weber ET, Gorlin R. Remission of cardiac lymphoma with supervoltage radiation. Am f Med 1971; 50: 816-22.

Requests for reprints to Dr I D A Johnston, St Thomas' Hospital, Lambeth Palace Road, London SE1. 